\title{
ASTR0N0MISCHE NACHRICHTEN.
}

№ 3058 .

\section{Der Photochronograph angewandt auf Doppelsternmessungen.}

\author{
Von $\mathcal{F}$. G. Hagen S. J.
}

\section{Einleitende Bemerkungen.}

1. In einer jüngst erschienenen Veröffentlichung des Georgetown Observatory wurde ein kleines Instrument beschrieben, das den in der Ueberschrift angegebenen Namen insofern verdient, als es auf der photographischen Platte das Bild eines gewöhnlichen Chronographen-Streifens verzeichnet, freilich mit dem wesentlichen Unterschiede, dass die Uhrsignale mit den gleichzeitigen Stellungen des Sternbildes identisch sind, dass also alle willkürlichen, mit persönlichen Fehlern behafteten Signale des Beobachters in Wegfall kommen.

Ganz zufällig hat sich nun herausgestellt, dass die durch diesen Apparat hervorgebrachten Bilder nicht nur zu Zeitbestimmungen, sondern auch zur Ausmessung von Doppelsternen geeignet sind. Der Stern $\zeta$ Ursae Majoris wurde von Prof. Fargis S. J., dem Erfinder des Apparats, zum Zwecke der Rectascensionsbestimmung photographirt, und zeigte unter dem Mikroskope nicht nur den mehr nördlich gelegenen Stern Alcor, sondern auch den südlichen Begleiter. Der Gedanke, wenigstens versuchsweise eine Ausmessung des Doppelsterns vorzunehmen, lag somit nahe, und um jedem Leser sein eigenes Urtheil über die Methode zu ermöglichen, lassen wir hier die Ergebnisse etwas ausführlich folgen.

Der Stern Mizar scheint in Folge seiner günstigen Stellung und Helligkeit allen neuen Methoden die erste Probe zu bieten. Nach Flammarion war er überhaupt der zuerst entdeckte teleskopische Doppelstern, da ihn Riccioli um I $65^{\circ}$ gesehen haben soll. Die deutschen Namen "Pferd", *Füllen * und Reiter scheinen den drei Sternen zuerst von Gottfried Kirch (1700) gegeben worden zu sein (siehe Bode's Jahrbuch für 1812, S. 252). Derselbe Doppelstern war auch der erste, der nach der gewöhnlichen Methode photographirt und ausgemessen wurde, woruber sich in den A. N. Bd. 47-49 (1858-1859) längere Abhandlungen Bond's finden. Weiter bildet Mizar den Gegenstand einer der schönsten Entdeckungen der Spectralphotographie, wie Pickering in dem vierten Jahresberichte des Henry Draper Memorial des weiteren beschrieben hat. Aus der Verdoppelung der $K$-Linie $\left(=H_{2}\right)$ in Zwischenräumen von $5^{2}$ Tagen schliesst nämlich Pickering, dass der Hauptstern ein unsichtbarer Doppelstern mit elliptischer Bahn ist, wenn auch nach Prof. Vogel (A.N. 30I7) , die Verhältnisse dieses Systems als noch nicht ganz aufgeklärt angesehen werden müssen *.

Wir erwähnen im Anschlusse an diese Entdeckung eine Bemerkung Pritchard's (A. N. 3037, S. 207), der es Bd. 128 sehr bedauert, dass seine photographische Methode zur Bestimmung der Sternparallaxen nicht auch auf Mizar Anwendung findet, eben weil das Spectrum dieses Sterns einen Doppelstern verräth, aus dessen Parallaxe man dann auf die scheinbare Entfernung der beiden Begleiter schliessen könnte. Der Uebelstand besteht nach Pritchard darin, dass die Bilder der zwei hellen um 14" von einander entfernten Componenten in einander verschwimmen und unmessbar werden, wenn man die Belichtungszeit lange genug ausdehnt, um auch die schwächeren Vergleichsterne auf der Platte zu erhalten. Diesem Uebelstande könnte nun durch das hier besprochene Instrument abgeholfen werden, indem sich diese beiden hellen Sterne beliebig lange verdunkeln lassen. Bei unserem Uhrcontacte geschieht die Verdunkelung während $\%$ der ganzen Expositionszeit, sie kann aber auch durch einen (in der erwähnten Schrift beschriebenen) Umschalter beliebig lange ausgedehnt werden, ja, der Stern kann von der photographischen Platte völlig ausgeschlossen werden. Um diese Abschwächung der aktinischen Strahlen auf einen beliebigen Stern aanzuwenden, müsste nur der Photochronograph wie ein Positionsmikrometer sich drehen und die Zunge durch ein bewegliches Knie sich biegen lassen. Allerdings verliert dann das Instrument, während das Aequatoreal durch Uhrwerk dem Sterne folgt, seinen ursprünglichen Zweck der Zeitregistrirung, indem alle Bilder des Sterns auf einander zu liegen kommen. Darin liegt aber gerade ein Fingerzeig, dass dieser Apparat eine Art Universalinstrument ist, das sich bei allen photographischen Aufnahmen nutzbar zu machen verspricht.

2. Es möge hier gleich von vornherein auf einen wesentlichen Unterschied der oben erwähnten Methoden von Doppelsternmessungen hingewiesen werden. Während man bei unmittelbarer Beobachtung durch das Auge nach längerem Betrachten des flackernden Sternbildes eine mittlere Lage des Bildes zu schätzen hat, findet man auf der Platte, welche dem Stern mittelst Uhrwerk folgt, alle die verschiedenen durch Unruhe der Luft verzerrten Bilder über einander gelagert, > eine selbstregistrirte mittlere Wirkung aller Störungen des Bildes ‘, wie Bond (A.N. I I29, S. 14) sich ausdrückt. Beide Methoden kommen aber darin überein, dass sie die Grösse dieser Schwankungen, gleichviel ob nacheinander oder gleichzeitig gesehen, nicht zu messen erlauben. Dieses leistet nun die dritte Methode des Photochronographen durch eine Reihe von Momentanbildern. Die Ausmessung dieser Bilder liefert nicht nur, wie die früheren Methoden, ein von atmosphärischen Störungen befreites mittleres Ergebniss, sondern auch ein getreues Bild dieser 
Störungen selbst, gleichsam, um uns des Kirch'schen Vergleichs zu bedienen, den ganzen Lauf von >Pferd s und "Fullen* durch das Gesichtsfeld in allen seinen Phasen.

Es wird sich herausstellen, dass diese Unregelmässigkeiten der Momentanbilder die zufälligen Fehler der Messung um mehr als das Doppelte übertreffen. Um nicht zu weitläufig zu werden, sollen dieselben durch ihr arithmetisches Mittel dargestellt werden, mit Angabe der grössten Ausschläge.

3. Wir geben zunächst eine Beschreibung der Platte. Der Doppelstern $\zeta$ Ursae Majoris ist $\Sigma_{1744}$ und gehört zu den helleren der VI. Ordnung Struve's, während er in Herschel's Synopsis als H III 2 bezeichnet ist. Die photographische Aufnahme geschah am 9. Mai 1891, an einem 40 Jahre alten Ertel'schen Durchgangsinstrumente von I I 5 Millimeter Oeffnung. Das photographische Bild selbst misst 19 Millimeter in der Länge und stellt einen Zeitraum von 4 Minuten dar, also eine Reihe von $4 \times 60$ Momentanbildern für jeden der beiden Begleiter, mit grösseren Unterbrechungen zwischen den einzelnen Minuten und einer kleineren an Stelle der 29. Zeitsecunde, wo keine Bilder entstehen. Diese Unterbrechungen sind wichtig, um die zusammengehörigen Bilder der beiden Begleiter ztl erkennen.

Die folgende Figur giebt einen allgemeinen Begriff der Platte, ohne Rücksicht auf Grössen-rerhältnisse und Anzahl der Punkte. Alcor war ausser dem Bereiche der Zunge und beschrieb deshalb eine gerade Linie. der Punktreihe (parallel dem Aequator) und in der Richtung der Verbindungslinie der beiden Begleiter liegt, so ist der Positionswinkel

$$
P=90^{\circ}+T-W
$$

Der Werth von $T$ wurde dreimal am Anfange und dreimal am Ende jeder Messungsreihe bestimmt, beruht also für jedè der drei Gruppen auf je 6 Einstellungen. Diese Richtung blieb im Mikroskop immer horizontal. Die Lage der Augen schien auf die Winkelmessungen keinen Einfluss zu haben, wie mehrere Versuche zeigten. Es ist ja auch von vornherein anzunehmen, dass die Fehlerquellen bei Ausmessung eines photographischen Negativs andere sein werden als bei unmittelbarer Beobachtung am Sterne. Die folgende Tafel zeigt das Ergebniss von 98 einzelnen Einstellungen auf 74 verschiedene Bilder des Doppelsterns. Die Gruppe II ist zweimal gemessen.

\begin{tabular}{c|c|c|c|c|c}
\hline Gruppe & $90^{\circ}+T$ & $W$ & $P$ & $\begin{array}{c}\text { Zahl der } \\
\text { Bilder }\end{array}$ & $\begin{array}{c}\text { Mittel der } \\
\text { Fehler }\end{array}$ \\
\hline I & 180.658 & 310852 & 148.806 & I9 & 1.06 \\
II & 180.747 & 32.954 & 147.793 & 24 & 1.15 \\
& 179.667 & 30.471 & 149.196 & 24 & 1.03 \\
III & 181.005 & 31.997 & 149.008 & 31 & 1.49
\end{tabular}

Die Fehler der Einstellungen des Fadens auf dasselbe Bild sind von einer niedrigeren Ordnung als die in der Tafel angegebenen, von der Unruhe der Atmosphäre verursachten Abweichungen. So betrug das arithme. tische Mittel der Fehler von $W$, bei wiederholten Einstellungen auf dasselbe Bild des Doppel. sterns, $28^{\prime}=0^{\circ} .47$, und dasjenige für $T$ nur $2: 2$.

\section{Benegungsridhtang}

Alcor

Mizar

Begleiter

$\because \div: \div: \bullet \bullet: \div: \div$

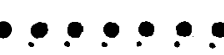

Von den vier Gruppen von Punktreihen, jede einer Zeitminute entsprechend, wurden nur die drei ersten als messbar befunden. Jede Gruppe fiel ganz innerhalb des Bereichs der Mikrometerschraube, aber nur etwa zwei Drittel der Punkte waren zur Messung scharf genug, so dass im Ganzen etwa 60 bis 70 Momentanbilder des Doppelsterns zur Verfügung standen. Die drei Gruppen wurden unabhängig von einander behandelt, um ein Maass für die innere Uebereinstimmung der Messungen zu haben, und mit I, II, III bezeichnet, in der Reihenfolge, wie sie durch die Bewegung des Sterns entstanden.

Wir schliessen hieran noch einige Bemerkungen über das Mikrometer. Dasselbe hat nur eine Schraube mit einem beweglichen Faden und ausserdem einen dazu senkrechten festen Faden, der aber bei der Ausmessung nicht zur Verwendung kam. Die zusammengehörigen Messungen wurden ohne Unterbrechung ausgeführt, und während dieser Operation wurden weder die photographische Platte noch die Stellschrauben des Centrirapparats berührt.

\section{Positions - Winkel.}

4. Sind $T$ und $W$ bezüglich die Ablesungen des Positionskreises, wenn der bewegliche Faden in der Richtung

Die grössten Abweichungen der einzelnen Sternbilder von ihrer mittleren Lage waren ungefähr $2.5 \mathrm{mal}$ so gross als. die in der Tafel angegebenen Mittel der Fehler.

Nimmt man die Zahl der gemessenen Bilder für die Gewichte $p$ der Gruppen, und vereinigt die beiden Messungen der II. Gruppe in ein Mittel, so erbält man folgenden wahrscheinlichen Werth des Positionswinkels mit den übrig bleibenden Fehlern $v$ :

\begin{tabular}{c|c|c}
\hline$P$ & $v$ & $p$ \\
\hline 148.806 & 0.089 & 19 \\
148.494 & 0.223 & 48 \\
149.008 & 0.291 & 31
\end{tabular}

$$
\begin{gathered}
P=148: 717 \pm 0.109 \text { d. h. } \pm 0.6745 \sqrt{\frac{5.162602}{2 \times 98}} \\
\text { oder } \pm 6: 56
\end{gathered}
$$

Der wabrscheinliche Fehler, ausgedrückt in Bogenmaass, ist :

$$
\pm \Delta \cdot \sin 6: 56= \pm 0.027
$$

also kaum die Hälfte desjenigen für die Distanzen, wie sich weiter unten zeigen wird. 


\section{Distanz.}

5. Wie in der erwähnten Veröffentlichung über den »Photochronographen « erwähnt wurde, liefern alle mit diesem Instrumente erhaltenen Platten unmittelbar den Schraubenwerth des Mikrometers, unabhängig von Aenderungen in der Brennweite des Fernrohrs oder dem Wärmezustande der Schraube. Es ist dieses Instrumentchen in der That ein selbstregistrirendes Mikrometer, das die hauptsächlichsten Aufgaben zu lösen verspricht, welche schon seit Jahren an die Himmesphotographie gestellt wurden (vergl. Dr. Lohse in den A. N. Bd. 115, S. 2 ).

Die Bestimmung einer Umdrehung der Mikrometerschraube für das vorliegende Negativ geschah in der in der genannten Schrift (p. 28) beschriebenen Weise. Alle Ablesungen $m$ der einzelnen Punkte und ihre entsprechenden Zeiten $t$ zwischen den Umdrehungen $0^{\mathbb{R}} \circ$ und $15^{\mathrm{R}}$. in arithmetische Mittel $M$ und $T$ vereinigt, ebenso die entsprechenden Werthe $m^{\prime}$ und $t^{\prime}$ zwischen den Umdrehungen $15^{\text {R.o }}$ und $30^{\mathbf{R}_{0}}$ in die Mittel $M^{\prime}$ und $T^{\prime}$. Der Werth einer Schraubenumdrehung ist dann

$$
x=\frac{T^{\prime}-T}{M^{\prime}-M} \cdot 15 \cos \delta, \quad\left(\delta=55^{\circ} 29^{\prime} 5\right) \text {. }
$$

Bei dieser Bestimmung wurden die fortschreitenden Fehler der Schraube in Rechnung gezogen, und von den beiden Punktreihen des Bildes nur diejenige des Hauptsterns benutzt. Auf jeden Punkt wurde nur einmal eingestellt.

Da jedes $T$ und $M$ dạs Mittel aus ungefähr 24 Einzelwerthen ist, so wurden den drei Gruppen gleiche Gewichte zugetheilt. Das Ergebniss ist folgendes :

\begin{tabular}{c|c|c|c}
\hline Gruppe & $x$ im Parallel & $x$ im Bogen & $v$ \\
\hline I & $1^{5} .7830$ & 15.1845 & 0.031 \\
II & 7805 & 1305 & 023 \\
III & 7823 & 1460 & 008 \\
\multicolumn{2}{c|}{$x=1^{5} .7819$ oder $15.1537 \pm 0.0108$}
\end{tabular}

Bei diesen Messungen war darauf gesehen, dass der bewegliche Faden senkrecht zur Richtung der Punktreihe stand. Zu diesem Zwecke wurde er erst dreimal auf diese Punktreihe gelegt und dann von dem Mittel der drei Ablesungen um 90 Grad gedreht, worauf der Positionskreis festgeklemmt wurde.

6. Nach Ausmessung des Schraubenwerths für diese Platte wurden die Abstände der beiden Sterne sofort bestimmt, ohne die Messungen zu unterbrechen. Die Richtung der Verbindungslinie beider Begleiter wurde aus dem oben angegebenen Werthe des Positionswinkels $P=\mathbf{1 4 8}_{42}$ bestimmt. Der bewegliche Faden wurde bei jeder Gruppe dreimal auf die Richtung der Punktreihe eingesetzt (früher mit $T$ bezeichnet) und dann, entsprechend dem gefundenen Positionswinkel, in die Verbindungslinie der beiden Sterne gedreht (nach der früheren Bezeichnung $T+W$ ).

Jedes Sternbild wurde mit dem beweglichen Faden nur einmal halbirt, so dass der Unterschied zweier zusammengehöriger Halbirungen eine Distanz-Messung darstellt. Diese einzelnen Messungen, innerhalb jeder Gruppe im Mittel vereinigt, lieferten das folgende Ergebniss:

\begin{tabular}{c|c|c|r}
\hline Gruppe & \multicolumn{1}{|c|}{$\boldsymbol{\Delta}$} & $\begin{array}{r}\text { Anzahl der } \\
\text { Doppelbilder }\end{array}$ & $\begin{array}{c}\text { Mittel der } \\
\text { Fehler }\end{array}$ \\
\hline I & 0.964 I & 17 & 0.485 \\
II & 9450 & 22 & 606 \\
III & 9616 & 23 & 546
\end{tabular}

Die Fehler der Einstellung sind auch hier, wie beim Positionswinkel, von einer niedrigeren Ordnung als die in der Tafel angeführten und hauptsächlich der Atmosphäre zuzuschreibenden Abweichungen. So betrug das arithmetische Mittel der Fehler bei wiederholtem Ausmessen desselben Bildes nur o." 24 .

Die grössten Abweichungen der einzelnen Distanzen 4 von den Mittelwerthen betragen in jeder Gruppe das Dreifache der in der Tafel angegebenen Abweichungen.

Nimmt man wieder die Zahl der gemessenen Doppelbilder für die Gewichte $p$ der drei Gruppen, so erhält man folgenden wahrscheinlichen Werth des Abstandes in Bogensecunden mit den übrig bleibenden Fehlern $v$ :

\begin{tabular}{r|r|r}
\hline \multicolumn{1}{c|}{$\Delta$} & $v$ & $p$ \\
\hline $14 \% 6097$ & 0.117 & 17 \\
3202 & 173 & 22 \\
5718 & 079 & 23
\end{tabular}

$\Delta=\mathrm{r} 4.493 \pm 0.062$ d. h. $\pm 0.6745 \sqrt{\frac{\mathrm{r.034694}}{2 \times 62}}$.

Es trifft sich zufällig, dass hier gerade so viele Bilder des Doppelsterns Mizar gemessen wurden, als Bond zur Verfügung standen, nämlich 62 (A. N. Nr. 1 129). Es wäre aber ein Irrthum, diese beiden Messungsreihen als gleichwerthig zu betrachten. Denn jede Platte Bond's folgte dem Sterne mittelst Uhrwerks und stellt daher schon das Mittel aller atmosphärischen Störungen während durchschnittlich 25 Secunden dar. Unsere Bilder dagegen hatten eine Belichtungszeit von einer Zehntel Secunde, also alle 62 Bilder eine solche von nur 6 Secunden. Die Unruhe der Sternbilder sollte also, wenn nur die Zeit in Betracht gezogen wird, auf einer einzigen Platte Bond's viermal besser eliminirt sein als in unseren drei Gruppen zusammenge. nommen.

7. Wir stellen hier die Ergebnisse mit ihren wahrscheinlichen Fehlern zusammen (1891 Mai 9):

$$
\begin{aligned}
& P=148.72 \pm 0.1 \mathrm{I} \text { (oder } \pm \Delta \cdot \sin 6: 6= \pm 0.03 \text { ) } \\
& \Delta=14.49 \pm 0.06 \text {. }
\end{aligned}
$$

Dunér's Elemente (Mes. Micrométriques, 1876 )

$$
\Delta=14: 29, \quad P=147^{\circ} 7+0.025(t-1855.0)
$$

lassen die folgenden Fehler übrig $(B-R):+0.20$ und -0 or.

Flammarion's Messungen (Catalogue, Paris 1878, p. 74) stimmen mit den oben mitgetheilten innerhalb der wahrscheinlichen Fehler überein :

$$
\Delta=14.55, \quad P=148.7 \text {. }
$$

Bond giebt in den A. N. I I05 die folgenden Werthe für I857: $\Delta=14: 49, \quad P=147^{\circ}{ }^{\circ}$, 
und in den A.N. 1129 bespricht er eine längere Reihe photographischer Aufnahmen dieses Doppelsterns. Er erhält aus 62 Platten die Entfernung $\Delta=14 \% 12$, giebt aber keinen Positionswinkel. Die wahrscheinlichen Fehler theilt er in drei Arten mit den folgenden Bezeichnungen :

$$
\begin{aligned}
& \varepsilon=\text { w. F. des photographischen Doppelbildes, } \\
& \varepsilon^{\prime}=>\text { des Mikrometers, } \\
& \varepsilon^{\prime \prime}=\text { einer Doppelbisection, }
\end{aligned}
$$

alle drei auf die Einheit der Gewichte bezogen. Er bezeichnet ferner mit $A, B, \ldots$ die Gewichte der einzelnen gemessenen Platten und findet für den Gesammtfehler einer Platte ( $n=$ Anzahl der Platten):

$$
\begin{aligned}
g & =0.6745 \sqrt{\frac{A v^{2}+B v^{\prime 2}+\cdots \cdot}{n-\mathbf{1}}} \\
& =\sqrt{\varepsilon^{2}+\varepsilon^{\prime 2}+\varepsilon^{\prime \prime 2}}= \pm \mathrm{r} .06 .
\end{aligned}
$$

Daraus bestimmt er dann nachträglich die einzelnen Fehler wie folgt:

$\varepsilon= \pm 0.072, \quad \varepsilon^{\prime}= \pm 0.063, \quad \varepsilon^{\prime \prime}= \pm 0.046$.

Struve (Mensurae Micr. Introd. p. LVIII) giebt bekanntlich für Doppelsterne dieser Ordnung (VI) und Helligkeit (A) als wahrscheinliche Fehler einer vollständigen Messung :

$$
\pm 0.127 \text { und } \pm 23: 9= \pm 0: 398
$$

oder $\pm 4 . \sin 24^{\prime}= \pm 0.097$

Bond vergleicht dann den relativen Werth einer seiner Platten mit einer Struve'schen Messung, indem er sein $\varepsilon$, mit Ausschluss der zufälligen Fehler des Mikroskops und der Bisection, dem Struve'schen Fehler \pm 0 ". 27 gegenüberstellt, und findet das Verhältniss $127^{2}: 7^{2}=3:$ I $\mathrm{zu}$ Gunsten seiner Methode.

Obwohl nun eine Vergleichung aller dieser wahrscheinlichen Fehler sehr zu Gunsten der photochronographischen Methode spricht, so möchten wir doch aus den Ergebnissen einer einzigen, nur zufällig erhaltenen Platte kein Werthverhältniss zwischen den drei Methoden ableiten.

\section{Rectascension und Declination.}

8. Bei Gelegenheit der Bestimmung des Schraubenwerthes durch die Abstände der aufeinanderfolgenden Bilder des Hauptsterns wurden zugleich auch die Rectascensionsunterschiede der beiden Begleiter gemessen. Das Ergebniss aus 73 Bildern des Doppelsterns, von denen jedes nur ein. mal eingestellt wurde, ist folgendes :

\begin{tabular}{c|c|c|c|c}
\hline Gruppe & $\Delta \alpha^{R}$ & $\Delta \alpha^{s}$ & $\Delta \alpha^{\prime \prime} \cdot \cos \delta$ & Bilder \\
\hline I & 0.5 I 34 & 0.9148 & $7 " 7799$ & I6 \\
II & 0.5047 & 0.8994 & 7.6484 & 39 \\
III & 0.5227 & 0.9314 & 7.9208 & I 8
\end{tabular}

Mittel $\quad \Delta a=0.9106 \pm 0.006 \mathrm{I}$

I $5 \Delta \alpha \cdot \cos \delta=7.744 \pm 0.052$.

Die fortschreitenden sowohl wie die periodischen Fehler der Schraube hoben sich bei diesen Differenzen vollständig auf, wie zu erwarten war.
Um diè Coordinaten $\imath u$ vervollständigen, wurden auch die Declinationsdifferenzen gemessen. Der bewegliche Faden wurde erst am Positionskreise dreimal auf die horizontale Punktreihe eingestellt, worauf der Kreis an der mittleren Ablesung festgeklemmt wurde. Dies geschah einzeln für jede Gruppe.

Die beiden parallelen Punktreihen wurden dann durch denselben beweglichen Faden in der Längenrichtung $5 \mathrm{mal}$ halbirt, und an alle Ablesungen wurden die fortschreitenden und periodischen Fehler der Schraube angebracht. Das Ergebniss ist folgendes :

\begin{tabular}{c|r|r}
\hline Gruppe & \multicolumn{1}{|c|}{$\Delta \delta$} & \multicolumn{1}{|c}{$v$} \\
\hline I & I 2:4366 & \multicolumn{1}{|c}{0.009} \\
II & 4654 & 020 \\
III & 4336 & 012
\end{tabular}

Mittel $\Delta \delta=12.4452 \pm 0.0067$

Eine Verbesserung für Refraction wurde nicht angebracht, da die Zenithdistanz nur $16^{\circ} 36^{\prime}$ beträgt.

Der wahrscheinliche Fehler von $\Delta \delta$ ist 8 bis $9 \mathrm{mal}$ kleiner als der von $\Delta \alpha$ und von $\Delta$, so dass die 5 malige Einstellung auf die ganze Punktreihe genauer ist als die einmalige Einstellung auf die einzelnen Punkte.

Wir haben also für die Coordinaten des Begleiters, bezogen auf den Hauptstern, die Werthe:

$$
\begin{aligned}
& \text { 1891 Mai 9: } \Delta a=+0.911 \pm 0.006 \\
& \Delta \delta=\text { - г2".445 } \pm 0.007 \text {. }
\end{aligned}
$$

9. Zur Vergleichung der verschiedenen Messungen unter einander, haben wir die beiden Formeln:

Für die Entfernung:

$$
\begin{aligned}
\Delta & =\sqrt{(\Delta \alpha \cdot 15 \cos \delta)^{2}+\Delta \delta^{2}} \\
& =\sqrt{7.744^{2}+12.445^{2}}=14.66 \\
\text { direct }: & 14: 49 \pm 0.06 ; \text { und }
\end{aligned}
$$

für den Positionswinkel :

$$
\begin{aligned}
& \frac{\Delta \delta}{\Delta \alpha \cdot 15 \cos \delta}=1.60699=\operatorname{tang} \lambda \\
& P=90^{\circ}+\lambda=14^{\circ} \mathrm{i} \mathrm{I} \\
& \text { direct: } 148: 72 \pm 0.11 \text {. }
\end{aligned}
$$

Wie man sieht, ist die innere Uebereinstimmung der einzelnen directen Messungsreihen grösser als die gegenseitige Uebereinstimmung verschiedener Messungsreihen, was auf das Vorhandensein von noch unberücksichtigten constanten Fehlern hindeutet.

Betrachtet man $\Delta \alpha$ als die unsicherste der vier gemessenen relativen Coordinaten, so scheint dieselbe nach beiden Vergleichungen etwas zu gross angenommen zu sein. Eine rückwärts angestellte Rechnung ergab für $\Delta \alpha$ aus dem Abstande $\Delta$ den Werth 7:42 76, und aus dem Positionswinkel $P$ den Werth $7 " 5618$, also im Mittel 7.495 oder $\Delta \alpha=0.88$ statt $0.9 \mathrm{r}$.

Man wird sich erinnern, dass auch Struve's Messungen von $\Delta \alpha$ durchschnittlich zu gross ausfielen (Mens. Micr., 
Introd. p. CXXXI). Doch lässt sich ein Zusammenhang dieser beiden Thatsachen nicht leicht absehen, da Struve's Erklärungsversuch auf die Ausmessung eines photographischen Negativs wohl keine Anwendung findet.

\section{Schlussbemerkungen.}

10. Die Durchmesser der Sternbilder wurden nur versuchsweise an 3 oder 4 Bildern gemessen und im Betrage von 5.4 und 4.0 bezüglich für Hauptstern und Begleiter gefunden. Die Intensität der Bilder hingegen ist so verschieden, dass man auf einen Unterschied von drei Grössenclassen schliessen möchte. Dembowski schätzt die Grössen zu 2 mo und $4 \%$, und die Farbe für den helleren Stern grünlich weiss, für den schwächeren dunkelgrün oder hellblau. Sestini S. J. hingegen schätzt in seiner Memoria (Roma 1845) Tav. II, No. 1534, beide Sterne weiss, den schwächeren vielleicht gelb.

Bond (A. N. I 158 ) hat die Durchmesser seiner Sternbilder als Function der Belichtungszeit dargestellt. Eine solche Untersuchung trifft bei der photochronographischen Methode nicht zu, da die Expositionszeiten für alle Sterne gleich sind (bei unserem Uhrcontact eine Zehntelsecunde). Aber gerade dieser Umstand dürfte bei Untersuchungen über die aktinische Kraft der verschiedenen Sterne, uber die Absorption der Luft und die Intensität der Platten von Bedeutung werden. Die auf dieser Sternwarte bis jetzt

Georgetown College Observatory, 189 I Juni. ausgemessenen vierhundert Platten zeigen in diesen Beziehungen manche unerwartete Erscheinungen, die einer späteren Untersuchung vorbehalten bleiben.

11. In der mehrerwähnten Abhandlung (A. N. I 129 , S. I3) bespricht Bond ausführlich die grossen Vortheile der photographischen Methode bei Doppelsternmessungen, namentlich die Beseitigung vieler persönlichen Fehler. Wir möchten noch einige woitere Vortheile erwähnen, die sich mehr auf die instrumentalen Fehler beziehen.

Die bekannte Erscheinung, dass in lichtschwachen Instrumenten die kleinen Distanzen fast immer zu gross gemessen werden (Mens. Micr. Introd., p. CXXXIV), indem die bei der angestrebten Bisection abgeschnittenen Segmente der Sternscheibchen gegen den Begleiter hin grösser ausfallen als die abgewandten, wird beim photographischen Negativ wohl kaum zutreffen.

Da weiter die photochronographische Methode ein in AR. festgeklemmtes Aequatoreal voraussetzt, so kann weder die Abweichung der. Polaraxe vom Pole noch auch die Unvollkommenheit der Triebuhr von schädlichem Einflusse sein.

Die mitgetheilten Messungen und Rechnungen dïrften vielleicht die Veranlassung sein, dass dieses kleine Instru. ment an irgend ein grosses Aequatoreal mit photographischer Linse angebracht wird. Es muss sich dann zeigen, bis auf welche Classe von Doppelsternen sich diese Methode ausdehnen lässt.

\section{Sonnenfleckenzählungen}

auf der Wiener Sternwarte ausgeführt von Herrn A. Kalbmayer.

Mitgetheilt von Prof. Dr. E. Weiss.

Die Zählungen der Sonnenflecken in II. Semester des Jahres 1890 wurden in derselben Weise ausgefuhrt, wie die im I. Semester, welche Astr. Nachr. Bd. I27 S. 39 mitgetheilt sind. Sie sind auch an demselben Fraunhofer'schen Fernrohre von $10^{\mathrm{cm}}$ Oeffnung bei 80 facher Vergrösserung mit einem neutralen Blendglase angestellt.

\begin{tabular}{|c|c|c|c|c|c|c|c|c|c|c|}
\hline \multicolumn{2}{|c|}{1890} & \multicolumn{3}{|c|}{ Zeit } & Gruppe & Flecken & Poren & $\begin{array}{r}\text { Isolirte } \\
\text { Flecken }\end{array}$ & $\begin{array}{l}\text { Isolirte } \\
\text { Poren }\end{array}$ & Bemerkungen \\
\hline \multirow[t]{12}{*}{ Juli } & 5 & $8^{h}$ & $30^{n}$ & a. m. & I & 2 & 2 & - & - & mit Fackeln $\mathbf{S}$ \\
\hline & 7 & 8 & 45 & 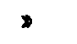 & $\mathbf{I}$ & 2 & 2 & $\mathbf{r}$ & 一 & $\mathbf{S} \mathbf{W}$ \\
\hline & 8 & 9 & 0 & 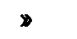 & 2 & 3 & $\mathbf{I}$ & 一 & 2 & $\mathbf{S} \mathbf{W}$ \\
\hline & 9 & 0 & 0 & > & 2 & 3 & $\mathbf{I}$ & - & 2 & WS W \\
\hline & I I & 8 & 30 & 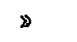 & $\mathbf{I}$ & $\mathbf{I}$ & 2 & - & - & $\mathrm{E}$ \\
\hline & I 6 & 8 & 30 & » & - & - & 一 & - & - & E gross. Fackelgeb. \\
\hline & 24 & ro & 45 & 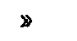 & $\mathbf{I}$ & 2 & $\mathbf{I}$ & - & 一 & W S W \\
\hline & 25 & 10 & 45 & ע & 2 & 3 & 2 & - & - & W S W \\
\hline & 26 & 8 & 45 & 》 & $\mathbf{r}$ & 2 & 3 & - & - & g. Mitte. \\
\hline & 27 & 9 & 0 & $»$ & $\mathbf{I}$ & 3 & I & - & - & Mitte. \\
\hline & 29 & 8 & 25 & $\gg$ & 2 & 4 & 2 & - & - & g. Mitte u. E mit Fackeln. \\
\hline & 31 & 8 & 35 & ๖ & 2 & - & - & $\mathbf{I}$ & 2 & W und g. E \\
\hline \multirow[t]{4}{*}{ Aug. } & $\mathbf{I}$ & 9 & 0 & » & $\mathbf{I}$ & 2 & $\mathbf{I}$ & - & I & \\
\hline & 2 & 8 & I 5 & $\searrow$ & $\mathbf{I}$ & 2 & 一 & - & I & W P. am Rand. \\
\hline & 3 & 8 & 0 & 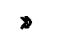 & $\mathbf{I}$ & 2 & - & - & - & Mitte. \\
\hline & 4 & 8 & 30 & » & $\mathbf{I}$ & I & $\mathbf{I}$ & - & - & Mitte. \\
\hline
\end{tabular}

